\title{
Forward integrals and an Itô formula for fractional Brownian motion
}

\author{
Francesca Biagini ${ }^{1)} \quad$ Bernt $\varnothing \mathrm{ksendal}^{2), 3)}$
}

Revised in April 2007*

1) Department of Mathematics, University of Bologna, Piazza di Porta S. Donato, 5

I-40127 Bologna, Italy

Email: biagini@dm.unibo.it

2) Center of Mathematics for Applications (CMA)

Department of Mathematics, University of Oslo

Box 1053 Blindern, N-0316 Oslo, Norway,

Email: oksendal@math.uio.no

3) Norwegian School of Economics and Business Administration,

Helleveien 30, N-5045 Bergen, Norway

\begin{abstract}
We consider the forward integral with respect to fractional Brownian motion $B^{(H)}(t)$ and relate this to the Wick-Itô-Skorohod integral by using the $M$-operator introduced by [10] and the Malliavin derivative $D_{t}^{(H)}$. Using this connection we obtain a general Itô formula for the Wick-Itô-Skorohod integrals with respect to $B^{(H)}(t)$, valid for $H \in\left(\frac{1}{2}, 1\right)$.
\end{abstract}

AMS 2000 subject classifications: Primary 60G15, 60G18, 60HXX, 60H07, $60 \mathrm{H} 40$.

Key words and phrases: Forward integral, Wick-Itô-Skorohod integral, Wick product, Malliavin derivative, fractional Brownian motion, Itô formula.

${ }^{*}$ Preprint of an article submitted for consideration in Infinite Dimensional Analysis, Quantum Probability and Related Topics 2008 [copyright World Scientific Publishing Company] [http://www.worldscinet.com/idaqp/idaqp.shtml] 


\section{Introduction}

Fractional Brownian motion $B^{(H)}(t)=B^{(H)}(t, \omega), t \geq 0, \omega \in \Omega$, with Hurst parameter $H \in(0,1)$ is a real-valued Gaussian process on a probability space $(\Omega, \mathcal{F}, \mathbb{P})$ with the property that

$$
E\left[B^{(H)}(t)\right]=B^{(H)}(0)=0 \text { for all } t \geq 0
$$

and

$$
E\left[B^{(H)}(t) B^{(H)}(s)\right]=\frac{1}{2}\left[t^{2 H}+s^{2 H}-|t-s|^{2 H}\right] ; \quad t, s \geq 0
$$

where $E$ denotes expectation with respect to $\mathbb{P}$.

Because of its properties the fractional Brownian motion has been used to model a number of phenomena, e.g. in biology, meteorology, physics and finance. See e.g. [24], [6], [7], [21] and the references therein. In that connection, it is of interest to develop a stochastic calculus based on $B^{(H)}(t)$. In particular, one wants an integration theory, a white noise theory and a Malliavin calculus for such processes. See e.g. [6] and the references therein for an account of this.

There are several different integral concepts of independent interest, among which the pathwise integral and the Wick-Itô-Skorohod integral. For each of these integrals several versions of an Itô formula have been obtained. See for example [5], [7], [9], [15], [18], [19], [11].

The purpose of this paper is to prove a new general Itô formula for the Wick-Itô-Skorohod integral based on the $M$-operator of [10] and the Malliavin derivative $D_{t}^{(H)}$, valid for $H \in\left(\frac{1}{2}, 1\right)$.

\section{Some preliminaries}

Here we recall the approach of [10], [16],[7] to white-noise calculus for fractional Brownian motion.

We begin by recalling the standard setup for the classical white noise probability space. See e.g. [13], [17], [14] or [1] for more details.

Definition 2.1 Let $\mathcal{S}(\mathbb{R})$ denote the Schwartz space of rapidly decreasing smooth functions on $\mathbb{R}$ and let $\Omega:=\mathcal{S}^{\prime}(\mathbb{R})$ be its dual, usually called the space of tempered distributions. Let $\mathbb{P}$ be the probability measure on the Borel sets $\mathcal{B}\left(\mathcal{S}^{\prime}(\mathbb{R})\right)$ defined by the property that

$$
\int_{\mathcal{S}^{\prime}(\mathbb{R})} \exp (i<\omega, f>) d \mathbb{P}(\omega)=\exp \left(-\frac{1}{2}\|f\|_{L^{2}(\mathbb{R})}^{2}\right) ; \quad f \in \mathcal{S}(\mathbb{R}),
$$


where $i=\sqrt{-1}$ and $\langle\omega, f\rangle=\omega(f)$ is the action of $\omega \in \Omega=\mathcal{S}^{\prime}(\mathbb{R})$ on $f \in \mathcal{S}(\mathbb{R})$.

The measure $\mathbb{P}$ is called the white noise probability measure. Its existence follows from the Bochner-Minlos theorem.

In the following we let

$$
h_{n}(x)=(-1)^{n} e^{\frac{x^{2}}{2}} \frac{d^{n}}{d x^{n}}\left(e^{-\frac{x^{2}}{2}}\right) ; \quad n=0,1,2, \ldots
$$

denote the Hermite polynomials and we let

$$
\xi_{n}(x)=\pi^{-\frac{1}{4}}((n-1) !)^{-\frac{1}{2}} h_{n-1}(\sqrt{2} x) e^{-\frac{x^{2}}{2}} ; \quad n=1,2, \ldots
$$

be the Hermite functions. Then $\xi_{n} \in \mathcal{S}(\mathbb{R})$. From [25], $\left\{\xi_{n}\right\}_{n=1}^{\infty}$ constitutes an orthonormal basis for $L^{2}(\mathbb{R})$. Let $\mathcal{J}$ be the set of all multi-indices $\alpha=\left(\alpha_{1}, \alpha_{2}, \ldots\right)$ of finite length $l(\alpha)=\max \left\{i ; \alpha_{i} \neq 0\right\}$, with $\alpha_{i} \in \mathbb{N}_{0}=$ $\{0,1,2, \ldots\}$ for all $i$. For $\alpha=\left(\alpha_{1}, \ldots, \alpha_{n}\right) \in \mathcal{J}$ we put $\alpha !=\alpha_{1} ! \alpha_{2} ! \cdots \alpha_{n}$ ! and $|\alpha|=\alpha_{1}+\cdots+\alpha_{n}$ and we define

$$
\mathcal{H}_{\alpha}(\omega)=h_{\alpha_{1}}\left(<\omega, \xi_{1}>\right) h_{\alpha_{2}}\left(<\omega, \xi_{2}>\right) \cdots h_{\alpha_{n}}\left(<\omega, \xi_{n}>\right) .
$$

In particular special cases are the unit vectors

$$
\epsilon^{(k)}=(0,0, \ldots, 0,1)
$$

with 1 on the $k$ 'th entry, 0 otherwise; $k=1,2, \ldots$. We now use the wellknown Wiener-Itô chaos expansion Theorem to define the following space $(\mathcal{S})$ of stochastic test functions and the dual space $(\mathcal{S})^{*}$ of stochastic distributions:

Definition 2.2 a) We define the Hida space (S) of stochastic test functions to be all $\psi \in L^{2}(\mathbb{P})$ whose expansion

$$
\psi(\omega)=\sum_{\alpha \in \mathcal{J}} a_{\alpha} \mathcal{H}_{\alpha}(\omega)
$$

satisfies

$$
\|\psi\|_{k}^{2}:=\sum_{\alpha \in \mathcal{J}} a_{\alpha}^{2} \alpha !(2 \mathbb{N})^{k \alpha}<\infty \quad \text { for all } k=1,2, \ldots
$$

where

$$
(2 \mathbb{N})^{\gamma}=(2 \cdot 1)^{\gamma_{1}}(2 \cdot 2)^{\gamma_{2}} \cdots(2 \cdot m)^{\gamma_{m}} \quad \text { if } \gamma=\left(\gamma_{1}, \ldots, \gamma_{m}\right) \in \mathcal{J}
$$


b) We define the Hida space $(\mathcal{S})^{*}$ of stochastic distributions to be the set of formal expansions

$$
G(\omega)=\sum_{\alpha \in \mathcal{J}} b_{\alpha} \mathcal{H}_{\alpha}(\omega)
$$

such that

$$
\|G\|_{q}^{2}:=\sum_{\alpha \in \mathcal{J}} b_{\alpha}^{2} \alpha !(2 \mathbb{N})^{-q \alpha}<\infty \quad \text { for some } q<\infty
$$

We equip (S) with the projective topology and $(\mathcal{S})^{*}$ with the inductive topology. Convergence in $(\mathcal{S})$ means convergence in $\|\cdot\|_{k}$ for every $k=$ $1,2, \cdots$, while convergence in $(\mathcal{S})^{*}$ means convergence in $\|\cdot\|_{q}$ for some $q<\infty$. Then $(\mathcal{S})^{*}$ can be identified with the dual of $(\mathcal{S})$ and the action of $G \in(\mathcal{S})^{*}$ on $\psi \in(\mathcal{S})$ is given by

$$
\langle G, \psi\rangle_{(\mathcal{S})^{*},(\mathcal{S})}:=\sum_{\alpha \in \mathcal{J}} \alpha ! a_{\alpha} b_{\alpha}
$$

In the sequel, we will denote the action $\langle\cdot, \cdot\rangle_{(\mathcal{S})^{*},(\mathcal{S})}$ simply with the symbol $\langle\cdot, \cdot\rangle$. We can in a natural way define $(\mathcal{S})^{*}$-valued integrals as follows:

Definition 2.3 (Integration in $\left.(\mathcal{S})^{*}\right)$ Suppose $Z: \mathbb{R} \rightarrow(\mathcal{S})^{*}$ has the property that

$$
\langle Z(t), \psi\rangle \in L^{1}(\mathbb{R}, d t) \quad \text { for all } \psi \in(\mathcal{S}) .
$$

Then the integral

$$
\int_{\mathbb{R}} Z(t) d t
$$

is defined to be the unique element of $(\mathcal{S})^{*}$ such that

$$
\left\langle\int_{\mathbb{R}} Z(t) d t, \psi\right\rangle=\int_{\mathbb{R}}\langle Z(t), \psi\rangle d t \quad \text { for all } \psi \in(\mathcal{S}) .
$$

Such functions $Z(t)$ are called $d t$-integrable in $(\mathcal{S})^{*}$.

Let $B(t)$ a standard Brownian motion on $(\Omega, \mathcal{F}, \mathbb{P})$. If we consider $B(t)$ as a map $B(\cdot): \mathbb{R} \rightarrow(\mathcal{S})^{*}$, then $B(t)$ is differentiable with respect to $t$ and its derivative $W(t):=\frac{d}{d t} B(t)$ exists in $(\mathcal{S})^{*}$ and is called white noise.

A fundamental property of the Wick product is the following relation to (Itô-)Skorohod integration. We recall the definition of Skorohod integral.

Let $u(t, \omega), \omega \in \Omega, t \in[0, T]$ be a stochastic process (always assumed to be $(t, \omega)$-measurable), such that

$$
u(t, \cdot) \quad \text { is } \mathcal{F} \text {-measurable for all } t \in[0, T]
$$


and

$$
E\left[u^{2}(t, \omega)\right]<\infty \quad \text { for all } t \in[0, T] .
$$

Definition 2.4 Suppose $u(t, \omega)$ is a stochastic process satisfying (2.11), (2.12) and with Wiener-Itô chaos expansion

$$
u(t, \omega)=\sum_{n=0}^{\infty} I_{n}\left(f_{n}(\cdot, t)\right)
$$

Then we define the Skorohod integral of $u$ by

$$
\delta(u):=\int_{\mathbb{R}} u(t, \omega) \delta B(t):=\sum_{n=0}^{\infty} I_{n+1}\left(\widetilde{f}_{n}\right) \quad \text { (when convergent) }
$$

where $\widetilde{f}_{n}$ is the symmetrization of $f_{n}\left(t_{1}, \ldots, t_{n}, t\right)$ as a function of $n+1$ variables $t_{1}, \ldots, t_{n}, t$.

We say $u$ is Skorohod-integrable and write $u \in \operatorname{dom}(\delta)$ if the series in (2.14) converges in $L^{2}(\mathbb{P})$. This occurs iff

$$
E\left[\delta(u)^{2}\right]=\sum_{n=0}^{\infty}(n+1) !\left\|\tilde{f}_{n}\right\|_{L^{2}\left(\mathbb{R}^{n+1}\right)}^{2}<\infty .
$$

Theorem 2.5 Suppose $f(t, \omega): \mathbb{R} \times \Omega \rightarrow \mathbb{R}$ is Skorohod integrable. Then $f(t, \cdot) \diamond W(t)$ is dt-integrable in $(\mathcal{S})^{*}$ and

$$
\int_{\mathbb{R}} f(t, \omega) \delta B(t)=\int_{\mathbb{R}} f(t, \omega) \diamond W(t) d t
$$

where the integral on the left is the Skorohod integral (which coincides with the Itô integral if $f$ is adapted) and $f(t, \omega) \diamond W(t)$ denotes the Wick product in $(\mathcal{S})^{*}$.

\subsection{Integration}

We now review briefly how the classical white noise theory can be used in order to construct a stochastic integral with respect to a fractional Brownian motion $B^{(H)}(t)$ for any $H \in(0,1)$ as in the approach of [10]. The main idea is to relate the fractional Brownian motion $B^{(H)}(t)$ with Hurst parameter $H \in(0,1)$ to classical Brownian motion $B(t)$ via the following operator $M$ : 
Definition 2.6 The operator $M=M^{(H)}$ is defined on functions $f \in \mathcal{S}(\mathbb{R})$ by

$$
\widehat{M f}(y)=|y|^{\frac{1}{2}-H} \hat{f}(y) ; \quad y \in \mathbb{R}
$$

where

$$
\hat{g}(y):=\int_{\mathbb{R}} e^{-i x y} g(x) d x
$$

denotes the Fourier transform.

For further details on the operator $M$, we refer to [10] and to [6]. The operator $M$ extends in a natural way from $\mathcal{S}(\mathbb{R})$ to the space

$$
\begin{aligned}
L_{H}^{2}(\mathbb{R}): & =\left\{f: \mathbb{R} \rightarrow \mathbb{R}(\text { deterministic }) ;|y|^{\frac{1}{2}-H} \hat{f}(y) \in L^{2}(\mathbb{R})\right\} \\
& =\left\{f: \mathbb{R} \rightarrow \mathbb{R} ; M f(x) \in L^{2}(\mathbb{R})\right\} \\
& =\left\{f: \mathbb{R} \rightarrow \mathbb{R} ;\|f\|_{L_{H}^{2}(\mathbb{R})}<\infty\right\}, \text { where }\|f\|_{L_{H}^{2}(\mathbb{R})}=\|M f\|_{L^{2}(\mathbb{R})} .
\end{aligned}
$$

The inner product on this space is

$$
\langle f, g\rangle_{L_{H}^{2}(\mathbb{R})}=\langle M f, M g\rangle_{L^{2}(\mathbb{R})}
$$

If $\left(\xi_{n}\right)_{n \in \mathbb{N}}$ is the orthonormal basis of $L^{2}(\mathbb{R})$ introduced in $(2.3)$, then

$$
e_{n}:=M^{-1} \xi_{n}, \quad \forall n \in \mathbb{N}
$$

is an orthonormal basis for $L_{H}^{2}(\mathbb{R})$. In particular, the indicator function $\chi_{[0, t]}(\cdot)$ is easily seen to belong to this space, for fixed $t \in \mathbb{R}$, and we write

$$
M \chi_{[0, t]}(x)=M[0, t](x) .
$$

We now define, for $t \in \mathbb{R}$

$$
\tilde{B}^{(H)}(t):=\tilde{B}^{(H)}(t, \omega):=<\omega, M[0, t](\cdot)>
$$

Then $\tilde{B}^{(H)}(t)$ is Gaussian, $\tilde{B}^{(H)}(0)=E\left[\tilde{B}^{(H)}(t)\right]=0$ for all $t \in \mathbb{R}$ and

$$
E\left[\tilde{B}^{(H)}(s) \tilde{B}^{(H)}(t)\right]=\frac{1}{2}\left[|t|^{2 H}+|s|^{2 H}-|s-t|^{2 H}\right]
$$

as follows by [10], (A.10). Therefore the continuous version of $B^{(H)}(t)$ of $\tilde{B}^{(H)}(t)$ is a fractional Brownian motion on $(\Omega, \mathcal{F}, \mathbb{P})$. Let $f \in L_{H}^{2}(\mathbb{R})$ and define

$$
\int_{\mathbb{R}} f(t) d B^{(H)}(t):=\int_{\mathbb{R}} M f(t) d B(t) ; \quad f \in L_{H}^{2}(\mathbb{R}) .
$$


Now define the fractional white noise $W^{(H)}(t)$ as the derivative with respect to $t$ of $B^{(H)}(t)$

$$
\frac{d B^{(H)}(t)}{d t}=W^{(H)}(t) \text { in }(\mathcal{S})^{*} .
$$

In particular, by [7] we obtain that the relation between fractional and classical white noise is given by

$$
W^{(H)}(t)=M W(t)
$$

In view of Theorem 2.5 the following definition is natural:

Definition 2.7 (The fractional Wick-Itô-Skorohod (WIS) integral) Let $Y: \mathbb{R} \rightarrow(\mathcal{S})^{*}$ be such that $Y(t) \diamond W^{(H)}(t)$ is dt-integrable in $(\mathcal{S})^{*}$. Then we say that $Y$ is $d B^{(H)}$-integrable and we define the Wick-Itô-Skorohod (WIS) integral of $Y(t)=Y(t, \omega)$ with respect to $B^{(H)}(t)$ by

$$
\int_{\mathbb{R}} Y(t, \omega) d B^{(H)}(t):=\int_{\mathbb{R}} Y(t) \diamond W^{(H)}(t) d t .
$$

Note that this definition coincides with $(2.22)$ if $Y=f \in L_{H}^{2}(\mathbb{R})$.

Definition 2.8 A process $Y(t)=\sum_{\alpha \in \mathcal{J}} c_{\alpha}(t) \mathcal{H}_{\alpha}(\omega) \in(\mathcal{S})^{*}$ belongs to the space $\mathcal{M}$ if $c_{\alpha}(\cdot) \in L_{H}^{2}(\mathbb{R})$ and $\sum_{\alpha \in \mathcal{J}} M c_{\alpha}(t) \mathcal{H}_{\alpha}(\omega)$ converges in $(\mathcal{S})^{*}$ for all $t$.

Then the following fundamental relation holds.

Proposition 2.9 (Integration) [BØSW, (5.2)], [Ø, (3.16)] Suppose $Y$ : $\mathbb{R} \rightarrow(\mathcal{S})^{*}$ is $d B^{(H)}$-integrable (Definition 2.7) and $Y \in \mathcal{M}$. Then

$$
\int_{\mathbb{R}} Y(t) d B^{(H)}(t)=\int_{\mathbb{R}} M Y(t) \delta B(t)
$$

\subsection{Differentiation}

We now recall the approach in [16] to differentiation, as modified and extended by [10]:

Definition 2.10 Let $F: \Omega \rightarrow \mathbb{R}$ and choose $\gamma \in \Omega$. Then we say $F$ has a directional $M$-derivative in the direction $\gamma$ if

$$
D_{\gamma}^{(H)} F(\omega):=\lim _{\varepsilon \rightarrow 0} \frac{1}{\varepsilon}[F(\omega+\varepsilon M \gamma)-F(\omega)]
$$

exists almost surely in $(\mathcal{S})^{*}$. In that case we call $D_{\gamma}^{(H)} F$ the directional $M$ derivative of $F$ in the direction $\gamma$. 
Definition 2.11 We say that $F: \Omega \rightarrow \mathbb{R}$ is differentiable if there exists a function

$$
\Psi: \mathbb{R} \rightarrow(\mathcal{S})^{*}
$$

in $\mathcal{M}$ such that

$$
D_{\gamma}^{(H)} F(\omega)=\int_{\mathbb{R}} M \Psi(t) M \gamma(t) d t \quad \text { for all } \quad \gamma \in L_{H}^{2}(\mathbb{R}) .
$$

Then we write

$$
D_{t}^{(H)} F:=\frac{\partial^{(H)}}{\partial \omega} F(t, \omega)=\Psi(t)
$$

and we call $D_{t}^{(H)} F$ the Malliavin derivative or the stochastic gradient of $F$ at $t$.

In the classical case $\left(H=\frac{1}{2}\right)$ we use the notation $D_{t}$ for the corresponding Malliavin derivative.

Proposition 2.12 [BØSW, (5.1)] Let $F \in(\mathcal{S})^{*}$. Then

$$
D_{t} F=M D_{t}^{(H)} F \quad \text { for a.a. } t \in \mathbb{R} .
$$

Proposition 2.13 [BØSW, Theorem 5.3] Suppose $Y: \mathbb{R} \rightarrow(\mathcal{S})^{*}$ is $d B^{(H)}$-integrable. If $D_{t} Y(\cdot): \mathbb{R} \rightarrow(\mathcal{S})^{*}$ is $d B^{(H)}$-integrable for every $t$, then

$$
D_{t}^{(H)}\left(\int_{\mathbb{R}} Y(s) d B^{(H)}(s)\right)=\int_{\mathbb{R}} D_{t}^{(H)} Y(s) d B^{(H)}(s)+Y(t) .
$$

Definition 2.14 Let $\mathbb{D}_{1,2}^{(H)}$ be the set of all $F \in L^{2}(\mathbb{P})$ such that the Malliavin derivative $D_{t}^{(H)} F$ exists and

$$
E\left[\int_{\mathbb{R}}\left[D_{t}^{(H)} F\right]^{2} d t\right]<\infty
$$

The following result has been obtained with a different proof in Lemma 2 of [18].

Lemma 2.15 Suppose $g \in L_{H}^{2}(\mathbb{R})$ and let $F \in \mathbb{D}_{1,2}^{(H)}$. Then

$$
F \diamond \int_{\mathbb{R}} g(t) d B^{(H)}(t)=F \cdot \int_{\mathbb{R}} g(t) d B^{(H)}(t)-\left\langle g, D^{(H)} F\right\rangle_{L_{H}^{2}(\mathbb{R})}
$$




\section{The forward integral}

By following the approach of [23], we now define the forward integral with respect to the fractional Brownian motion as follows:

\section{Definition 3.1}

a) The (classical) forward integral of a real valued measurable process $Y$ with integrable trajectories is defined by

$$
\int_{0}^{T} Y(t) d^{-} B^{(H)}(t)=\lim _{\epsilon \longrightarrow 0} \int_{0}^{T} Y(t) \frac{B^{(H)}(t+\epsilon)-B^{(H)}(t)}{\epsilon} d t,
$$

provided that the limit exists in probability under $\mathbb{P}$.

b) The (generalized) forward integral of a real valued measurable process $Y$ with integrable trajectories is defined by

$$
\int_{0}^{T} Y(t) d^{-} B^{(H)}(t)=\lim _{\epsilon \longrightarrow 0} \int_{0}^{T} Y(t) \frac{B^{(H)}(t+\epsilon)-B^{(H)}(t)}{\epsilon} d t,
$$

provided that the limit exists in $(\mathcal{S})^{*}$.

Note that in the generalized definition of forward integral, the limit is required to exist in the Hida space of stochastic distributions $(\mathcal{S})^{*}$ introduced in Definition 2.2. Convergence in $(\mathcal{S})^{*}$ is also explained in Section 2.

Corollary 3.2 Let $\psi(t)=\psi(t, \omega)$ be a measurable forward integrable process and assume that $\psi$ is càglàd. The forward integral of $\psi$ with respect to the fractional Brownian motion $B^{(H)}$ coincides with

$$
\int_{0}^{T} \psi(t) d^{-} B^{(H)}(t)=\lim _{|\Delta| \longrightarrow 0} \sum_{j=1}^{N} \psi\left(t_{j}\right) \Delta B_{t_{j}}^{(H)}
$$

whenever the left-hand limit exists in probability, where $\pi: 0=t_{0}<t_{1}<$ $\cdots<t_{N}=T$ is a partition of $[0, T]$ with mesh size $|\Delta|=\sup _{j=0, \cdots, N-1}\left|t_{j+1}-t_{j}\right|$ and $\Delta B_{t_{j}}^{(H)}=B_{t_{j+1}}^{(H)}-B_{t_{j}}^{(H)}$.

Proof. Let $\psi$ be a càglàd forward integrable process and

$$
\psi^{(\Delta)}(t)=\sum_{k} \psi\left(t_{k}\right) \chi_{\left(t_{k}, t_{k+1}\right]}(t)
$$


be a càglàd step function approximation to $\psi$. Then $\psi^{(\Delta)}(t)$ converges boundedly almost surely to $\psi(t)$ as $|\Delta| \longrightarrow 0$. The forward integral of $\psi^{(\Delta)}(t)$ is then given by

$$
\begin{aligned}
\int_{0}^{T} \psi^{(\Delta)}(t) d^{-} B^{(H)}(t) & =\lim _{\epsilon \rightarrow 0} \int_{0}^{T} \psi^{(\Delta)}(s) \frac{B^{(H)}(s+\epsilon)-B^{(H)}(s)}{\epsilon} d s \\
& =\lim _{\epsilon \rightarrow 0} \sum_{k} \psi\left(t_{k}\right) \int_{t_{k}}^{t_{k+1}} \frac{1}{\epsilon} \int_{s}^{s+\epsilon} d B^{(H)}(u) d s \\
& =\lim _{\epsilon \rightarrow 0} \sum_{k} \psi\left(t_{k}\right) \int_{t_{k}}^{t_{k+1}} \frac{1}{\epsilon} \int_{u-\epsilon}^{u} d s d B^{(H)}(u) \\
& =\sum_{k} \psi\left(t_{k}\right) \Delta B_{t_{k}}^{(H)}
\end{aligned}
$$

where $\Delta B_{t_{k}}^{(H)}=B_{t_{k+1}}^{(H)}-B_{t_{k}}^{(H)}$. Hence (3.1) follows by the dominated convergence theorem and by (3.3).

For the sequel we will use the same notation as in Section 2.

Definition 3.3 The space $\mathbb{L}_{1,2}^{(H)}$ consists of all càglàd processes

$$
\psi(t)=\sum_{\alpha \in \mathcal{J}} c_{\alpha}(t) \mathcal{H}_{\alpha}(\omega) \in(\mathcal{S})^{*}
$$

for every $t \in[0, T]$ and such that

$$
\|\psi\|_{\mathbb{L}_{1,2}^{(H)}}^{2}:=\sum_{\alpha \in \mathcal{J}} \sum_{i=1}^{\infty} \alpha_{i} \alpha !\left\|c_{\alpha}\right\|_{L^{2}([0, T])}^{2}<\infty .
$$

Note that if $\psi(t) \in(\mathcal{S})^{*}$ for every $t \in[0, T]$, then $D_{s} \psi(t)$ exists in $(\mathcal{S})^{*}$ (see Lemma 3.10 of [1]). We recall a preliminary lemma needed in the following.

Lemma 3.4 Let $(\Gamma, \mathcal{G}, m)$ be a measure space. Let $f_{\epsilon}: \Gamma \rightarrow B, \epsilon \in \mathbb{R}$, be measurable functions with values in a Banach space $\left(B,\|\cdot\|_{B}\right)$. If $f_{\epsilon}(\gamma) \rightarrow$ $f_{0}(\gamma)$ as $\epsilon \rightarrow 0$ for almost every $\gamma \in \Gamma$ and there exists $K<\infty$ such that

$$
\int_{\Gamma}\left\|f_{\epsilon}(\gamma)\right\|_{B}^{2} d m(\gamma)<K
$$

for all $\epsilon \in \mathbb{R}$, then

$$
\int_{\Gamma} f_{\epsilon}(\gamma) d m(\gamma) \rightarrow \int_{\Gamma} f_{0}(\gamma) d m(\gamma)
$$

in $\|\cdot\|_{B}$. 
Proof. The proof is analogous to the one of Theorem II.21.2 of [22].

Lemma 3.5 Suppose that $\psi \in \mathbb{L}_{1,2}^{(H)}$. Then

$$
M_{t+} D_{t+} \psi(t):=\lim _{\epsilon \longrightarrow 0} \frac{1}{\epsilon} \int_{t}^{t+\epsilon} M_{s} D_{s} \psi(t) d s
$$

exists in $L^{2}(\mathbb{P})$ for all $t$. Moreover

$$
\int_{0}^{T} M_{t+} D_{t+} \psi(t) d t=\lim _{\epsilon \longrightarrow 0} \int_{0}^{T}\left(\frac{1}{\epsilon} \int_{t}^{t+\epsilon} M_{s} D_{s} \psi(t) d s\right) d t
$$

in $L^{2}(\mathbb{P})$ and

$$
E\left[\left(\int_{0}^{T} M_{s+} D_{s+} \psi(s) d s\right)^{2}\right]<\infty
$$

Proof. Suppose that $\psi(t)$ has the expansion

$$
\psi(t)=\sum_{\alpha \in \mathcal{J}} c_{\alpha}(t) \mathcal{H}_{\alpha}(\omega)
$$

In the sequel we drop $\omega$ in $\mathcal{H}_{\alpha}(\omega)$ for the sake of simplicity. Then we have

$$
D_{s} \psi(t)=\sum_{\alpha \in \mathcal{J}} \sum_{i=1}^{\infty} c_{\alpha}(t) \alpha_{i} \mathcal{H}_{\alpha-\epsilon^{(i)}} \xi_{i}(s)
$$

and

$$
M_{s} D_{s} \psi(t)=\sum_{\alpha \in \mathcal{J}} \sum_{i=1}^{\infty} c_{\alpha}(t) \alpha_{i} \mathcal{H}_{\alpha-\epsilon^{(i)}} \eta_{i}(s),
$$

where $\eta_{i}(s)=M \xi_{i}(s)$. Hence

$$
\frac{1}{\epsilon} \int_{t}^{t+\epsilon} M_{s} D_{s} \psi(t) d s=\sum_{\alpha \in \mathcal{J}} \sum_{i=1}^{\infty}\left(c_{\alpha}(t) \frac{1}{\epsilon} \int_{t}^{t+\epsilon} \eta_{i}(s) d s\right) \alpha_{i} \mathcal{H}_{\alpha-\epsilon} .
$$

Since $\eta_{i}(s)=M \xi(s)$ is a continuous function, we have that

$$
\frac{1}{\epsilon} \int_{t}^{t+\epsilon} \eta_{i}(s) d s \rightarrow \eta_{i}(t)
$$

as $\epsilon \rightarrow 0$.

We apply now Lemma 3.4 with $\gamma=(\alpha, i), d m(\gamma)=\sum_{\alpha \in \mathcal{J}} \sum_{i=1}^{\infty} \delta_{(\alpha, i)}$, where 
$\delta_{x}$ denotes the point mass at $x, B=L^{2}(\mathbb{P})$ and $f_{\epsilon}=\left(c_{\alpha}(t) \frac{1}{\epsilon} \int_{t}^{t+\epsilon} \eta_{i}(s) d s\right) \alpha_{i} \mathcal{H}_{\alpha-\epsilon^{(i)}}$. We obtain

$$
\begin{aligned}
\int_{\Gamma}\left\|f_{\epsilon}(\gamma)\right\|_{B}^{2} d m(\gamma) & =\sum_{\alpha \in \mathcal{J}} \sum_{i=1}^{\infty}\left\|f_{\epsilon}(\gamma)\right\|_{L^{2}(\mathbb{P})}^{2} \\
& =\sum_{\alpha \in \mathcal{J}} \sum_{i=1}\left(c_{\alpha}(t) \frac{1}{\epsilon} \int_{t}^{t+\epsilon} \eta_{i}(s) d s\right)^{2} \alpha_{i} \alpha ! \\
& \leq\left[\frac{(t+\epsilon)^{2 H}-t^{2 H}}{\epsilon}\right]^{2} \sum_{\alpha \in \mathcal{J}} \sum_{i=1} c_{\alpha}(t)^{2} \alpha_{i} \alpha !
\end{aligned}
$$

since

$$
\begin{array}{r}
\frac{1}{\epsilon} \int_{t}^{t+\epsilon} \eta_{i}(s) d s=\left\langle M \xi_{i}, \frac{1}{\epsilon} \chi_{[t, t+\epsilon]}\right\rangle_{L^{2}(\mathbb{R})}= \\
\left\langle M^{2} e_{i}, \frac{1}{\epsilon} \chi_{[t, t+\epsilon]}\right\rangle_{L^{2}(\mathbb{R})}=\left\langle e_{i}, \frac{1}{\epsilon} \chi_{[t, t+\epsilon]}\right\rangle_{L_{H}^{2}(\mathbb{R})} \leq \\
\left\|e_{i}\right\|_{L_{H}^{2}(\mathbb{R})} \frac{1}{\epsilon}\left\|\chi_{[t, t+\epsilon]}\right\|_{L_{H}^{2}(\mathbb{R})}=\frac{(t+\epsilon)^{2 H}-t^{2 H}}{\epsilon},
\end{array}
$$

where we have used that the fact that $\left\|e_{i}\right\|_{L_{H}^{2}(\mathbb{R})}=1$ and the equality

$$
\int_{\mathbb{R}}[M[a, b](x)]^{2} d x=(b-a)^{2 H} .
$$

Since we have $\sum_{\alpha \in \mathcal{J}} \sum_{i=1} c_{\alpha}(t)^{2} \alpha_{i} \alpha !<\infty$ for almost every $t$, by Lemma 3.4 it follows that $\sum_{\alpha \in \mathcal{J}} \sum_{i=1}^{\infty}\left(c_{\alpha}(t) \frac{1}{\epsilon} \int_{t}^{t+\epsilon} \eta_{i}(s) d s\right) \alpha_{i} \mathcal{H}_{\alpha-\epsilon^{(i)}}$ converges to

$$
\sum_{\alpha \in \mathcal{J}} \sum_{i=1}^{\infty} c_{\alpha}(t) \eta_{i}(t) \alpha_{i} \mathcal{H}_{\alpha-\epsilon^{(i)}}
$$

in $L^{2}(\mathbb{P})$.

We now prove (3.8). Consider

$\int_{0}^{T} \frac{1}{\epsilon} \int_{t}^{t+\epsilon} M_{s} D_{s} \psi(t) d s d t=\sum_{\alpha \in \mathcal{J}} \sum_{i=1}^{\infty} \int_{0}^{T}\left(c_{\alpha}(t) \frac{1}{\epsilon} \int_{t}^{t+\epsilon} \eta_{i}(s) d s\right) d t \alpha_{i} \mathcal{H}_{\alpha-\epsilon^{(i)}}$.

Now assuming $f_{\epsilon}=\int_{0}^{T}\left(c_{\alpha}(t) \frac{1}{\epsilon} \int_{t}^{t+\epsilon} \eta_{i}(s) d s\right) d t \alpha_{i} \mathcal{H}_{\alpha-\epsilon^{(i)}}$ and as before $\gamma=$ $(\alpha, i), B=L^{2}(\mathbb{P}), d m(\gamma)=\sum_{\alpha \in \mathcal{J}} \sum_{i=1}^{\infty} \delta_{\alpha, i}$, where $\delta_{x}$ denotes the point mass 
at $x$, we use again Lemma 3.4. We obtain

$$
\begin{aligned}
\int_{\Gamma}\left\|f_{\epsilon}(\gamma)\right\|_{B}^{2} d m(\gamma) & =\sum_{\alpha \in \mathcal{J}} \sum_{i=1}^{\infty}\left\|f_{\epsilon}(\gamma)\right\|_{L^{2}(\mathbb{P})}^{2} \\
& =\sum_{\alpha \in \mathcal{J}} \sum_{i=1}\left(\int_{0}^{T} c_{\alpha}(t) \frac{1}{\epsilon} \int_{t}^{t+\epsilon} \eta_{i}(s) d s d t\right)^{2} \alpha_{i} \alpha ! \\
& \leq \sum_{\alpha \in \mathcal{J}} \sum_{i=1}\left(\int_{0}^{T} c_{\alpha}(t)\left[\frac{(t+\epsilon)^{2 H}-t^{2 H}}{\epsilon}\right] d t\right)^{2} \alpha_{i} \alpha ! \\
& \leq \sum_{\alpha \in \mathcal{J}} \sum_{i=1}\left(\int_{0}^{T} c_{\alpha}(t)^{2} d t\right)\left(\int_{0}^{T}\left[\frac{(t+\epsilon)^{2 H}-t^{2 H}}{\epsilon}\right]^{2} d t\right) \alpha_{i} \alpha !
\end{aligned}
$$

Since $\psi \in \mathbb{L}_{1,2}^{(H)}$ by Lemma 3.4 we can conclude that the limit 3.8 exists in $L^{2}(\mathbb{P})$ and also that $(3.9)$ holds.

Lemma 3.6 Suppose that $\psi \in \mathbb{L}_{1,2}^{(H)}$ and let

$$
\psi^{(\Delta)}(s)=\sum_{k} \psi\left(t_{k}\right) \chi_{\left(t_{k}, t_{k+1}\right]}(s)
$$

be a càglàd step function approximation to $\psi$, where $\Delta=\max _{i}\left|\Delta t_{i}\right|$ is the maximal length of the subinterval in the partition $0=t_{0}<\cdots<t_{n}=T$ of $[0, T]$. Then $\psi^{(\Delta)} \in \mathbb{L}_{1,2}^{(H)}$ for all $\Delta$ and

$$
\int_{0}^{T} M_{s+} D_{s+} \psi^{(\Delta)}(s) d s \longrightarrow \int_{0}^{T} M_{s+} D_{s+} \psi(s) d s \quad \text { in } L^{2}(\mathbb{P})
$$

as $|\Delta| \longrightarrow 0$.

Proof. $\quad$ Since $\psi^{(\Delta)}(s)=\sum_{\alpha \in \mathcal{J}} c_{\alpha}^{(\Delta)}(s) \mathcal{H}_{\alpha}(\omega)$ with

$$
c_{\alpha}^{(\Delta)}(s)=\sum_{k} c_{\alpha}\left(t_{k}\right) \chi_{\left(t_{k}, t_{k+1}\right]}(s)
$$

and

$$
\left\|c_{\alpha}^{(\Delta)}\right\|_{L^{2}([0, T])} \leq \text { const. }\left\|c_{\alpha}\right\|_{L^{2}([0, T])} \quad \forall \alpha
$$


it follows that $\psi^{(\Delta)} \in \mathbb{L}_{1,2}^{(H)}$. We have

$$
\frac{1}{\epsilon} \int_{t}^{t+\epsilon} M_{s} D_{s} \psi^{(\Delta)}(t) d s=\sum_{\alpha \in \mathcal{J}} \sum_{i=1}^{\infty}\left(\int_{0}^{T}\left(c_{\alpha}^{(\Delta)}(t) \frac{1}{\epsilon} \int_{t}^{t+\epsilon} \eta_{i}(s) d s\right) d t\right) \alpha_{i} \mathcal{H}_{\alpha-\epsilon}(i) .
$$

If we assume $\gamma=(\alpha, i), B=L^{2}(\mathbb{P}), m(d \gamma)=\sum_{\alpha \in \mathcal{J}} \sum_{i=1}^{\infty} \delta_{(\alpha, i)}$, where $\delta_{x}$ denotes the point mass at $x$, and $\left.f_{\Delta}=\left(\int_{0}^{T} c_{\alpha}^{(\Delta)}(t) \frac{1}{\epsilon} \int_{t}^{t+\epsilon} \eta_{i}(s) d s\right) d t\right) \alpha_{i} \mathcal{H}_{\alpha-\epsilon^{(i)}}$, with the same argument as in (3.10) by Lemma 3.4 we obtain that

$$
\int_{0}^{T}\left(\frac{1}{\epsilon} \int_{t}^{t+\epsilon} M_{s} D_{s} \psi(t) d s\right) d t=\lim _{|\Delta| \longrightarrow 0} \int_{0}^{T}\left(\frac{1}{\epsilon} \int_{t}^{t+\epsilon} M_{s} D_{s} \psi^{(\Delta)}(t) d s\right) d t
$$

in $L^{2}(\mathbb{P})$ for almost every $s$, since $c_{\alpha}^{(\Delta)}$ converges by dominated convergence to $c_{\alpha}$ in $L^{2}(\mathbb{P})$ and $\psi^{(\Delta)} \in \mathbb{L}_{1,2}^{(H)}$. Using (3.14) and Lemma 3.5 we conclude that (3.12) holds.

We now investigate the relation among forward integrals and WIS-integrals for $H>\frac{1}{2}$.

In [4] and [19] a similar relation is established between the symmetric integral and the divergence, in [9] between the forward integral and the fractional Wick-Itô-Skorohod integral. For the case $H<\frac{1}{2}$, we refer to [2].

Theorem 3.7 Let $H \in(0,1)$. Suppose $\psi \in \mathbb{L}_{1,2}^{(H)}$ and that one of the following conditions holds:

i) $\psi$ is Wick-Itô-Skorohod integrable (Definition 2.7);

ii) $\psi$ is forward integrable in $(\mathcal{S})^{*}$ (Definition 3.1).

Then

$$
\int_{0}^{T} \psi(t) d^{-} B^{(H)}(t)=\int_{0}^{T} \psi(t) d B^{(H)}(t)+\int_{0}^{T} M_{t+} D_{t+} \psi(t) d t,
$$

holds as an identity in $(\mathcal{S})^{*}$, where here $\int_{0}^{T} \psi(t) d B^{(H)}(t)$ is the WIS-integral of Definition 2.7.

Proof. We prove (3.15) assuming that hypothesis $i$ ) is in force. The argument works symmetrically under hypothesis $i i)$. Let $\psi \in \mathbb{L}_{1,2}^{(H)}$. Since $\psi$ is càglàd, we can approximate it as

$$
\psi(t)=\lim _{|\Delta t| \longrightarrow 0} \sum_{j} \psi\left(t_{j}\right) \chi_{\left(t_{j}, t_{j+1}\right]}(t) \quad \text { a.e. }
$$


where for any partition $0=t_{0}<t_{1}<\cdots<t_{N}=T$ of $[0, T]$, with $\Delta t_{j}=$ $t_{j+1}-t_{j}$, we have put $|\Delta t|=\sup _{j=0, \cdots, N-1} \Delta t_{j}$.

As before we put $\psi^{(\Delta)}(t)=\sum_{j=0}^{N-1} \psi\left(t_{k}\right) \chi_{\left(t_{k}, t_{k+1}\right]}(t)$ and evaluate

$$
\begin{array}{r}
\int_{0}^{T} \psi^{(\Delta)}(t) d^{-} B^{(H)}(t)=\lim _{\epsilon \rightarrow 0} \int_{0}^{T} \psi^{(\Delta)}(t, \omega) \frac{B^{(H)}(t+\epsilon)-B^{(H)}(t)}{\epsilon} d t= \\
\lim _{\epsilon \rightarrow 0} \int_{0}^{T}\left(\sum_{j} \psi\left(t_{j}\right) \chi_{\left(t_{j}, t_{j+1}\right]}(t)\right) \frac{1}{\epsilon} \int_{t}^{t+\epsilon} d B^{(H)}(u) d t= \\
\lim _{\epsilon \rightarrow 0} \int_{0}^{T}\left(\sum_{j} \psi\left(t_{j}\right) \chi_{\left(t_{j}, t_{j+1}\right]}(t)\right) \diamond \frac{1}{\epsilon} \int_{t}^{t+\epsilon} d B^{(H)}(u) d t+ \\
\lim _{\epsilon \rightarrow 0} \sum_{j} \int_{0}^{T} \chi_{\left(t_{j}, t_{j+1}\right]}(t) \frac{1}{\epsilon} \int_{\mathbb{R}} \chi_{[t, t+\epsilon]}(u) M_{u}^{2} D_{u}^{(H)} \psi\left(t_{j}\right) d u d t .
\end{array}
$$

The first limit is equal to

$$
\begin{array}{r}
\lim _{\epsilon \rightarrow 0} \int_{0}^{T}\left(\sum_{j} \psi\left(t_{j}\right) \chi_{\left(t_{j}, t_{j+1}\right]}(t)\right) \diamond \frac{1}{\epsilon} \int_{t}^{t+\epsilon} d B^{(H)}(u) d t= \\
\lim _{\epsilon \rightarrow 0} \int_{0}^{T}\left(\sum_{j} \psi\left(t_{j}\right) \chi_{\left(t_{j}, t_{j+1}\right]}(t)\right) \diamond \frac{1}{\epsilon} \int_{t}^{t+\epsilon} W^{(H)}(u) d u d t= \\
\lim _{\epsilon \rightarrow 0} \int_{0}^{T} \frac{1}{\epsilon}\left(\int_{u-\epsilon}^{u} \sum_{j} \psi\left(t_{j}\right) \chi_{\left(t_{j}, t_{j+1}\right]}(t)\right) \diamond W^{(H)}(u) d u= \\
\int_{0}^{T} \psi^{(\Delta)}(u) \diamond W^{(H)}(u) d u,
\end{array}
$$

that converges in $(\mathcal{S})^{*}$ to $\int_{0}^{T} \psi(u) \diamond W^{(H)}(u) d u=\int_{0}^{T} \psi(u) d B^{(H)}(u)$. For the second limit we get

$$
\begin{array}{r}
\lim _{\epsilon \rightarrow 0} \frac{1}{\epsilon} \sum_{j} \int_{0}^{T} \chi_{\left(t_{j}, t_{j+1}\right]}(t) \int_{t}^{t+\epsilon} M_{u}^{2} D_{u}^{(H)} \psi\left(t_{j}\right) d u d t= \\
\lim _{\epsilon \rightarrow 0} \int_{0}^{T} \frac{1}{\epsilon} \int_{t}^{t+\epsilon} M_{u}^{2} D_{u}^{(H)} \psi^{(\Delta)}(t) d u d t= \\
\lim _{\epsilon \longrightarrow 0} \int_{0}^{T} \frac{1}{\epsilon} \int_{t}^{t+\epsilon} M_{u} D_{u} \psi^{(\Delta)}(t) d u d t .
\end{array}
$$

By Lemmas 3.5 and 3.6 the last limit converges to

$$
\int_{0}^{T} M_{u+} D_{u+} \psi(u) d u
$$


in $L^{2}(\mathbb{P})$.

An analogous relation to the one of Theorem 3.7 between Stratonovich integrals and Wick-Itô-Skorohod integrals for fractional Brownian motion is proved under different conditions in [18].

An Itô formula for forward integrals with respect to classical Brownian motion was obtained by [23] and then extended to the fractional Brownian motion case in [12]. Here we prove the following Itô formula for forward integrals with respect to fractional Brownian motion as a consequence of Lemma 3.8 .

Lemma 3.8 Let $G \in(\mathcal{S})^{*}$ and suppose that $\psi$ is forward integrable. Then

$$
G(\omega) \int_{0}^{T} \psi(t) d^{-} B^{(H)}(t)=\int_{0}^{T} G(\omega) \psi(t) d^{-} B^{(H)}(t)
$$

Proof. This is immediate by Definition 3.1.

Definition 3.9 Let $\psi$ be a forward integrable process and let $\alpha(s)$ be a measurable process such that $\int_{0}^{t}|\alpha(s)| d s<\infty$ a.s. for all $t \geq 0$. Then the process

$$
X(t):=x+\int_{0}^{t} \alpha(s) d s+\int_{0}^{t} \psi(s) d^{-} B^{(H)}(s) ; \quad t \geq 0
$$

is called a fractional forward process. As a shorthand notation for (3.18) we write

$$
d^{-} X(t):=\alpha(t) d t+\psi(t) d^{-} B^{(H)}(t) ; \quad X(0)=x .
$$

Theorem 3.10 Let

$$
d^{-} X(t)=\alpha(t) d t+\psi(t) d^{-} B^{(H)}(t) ; X(0)=x
$$

be a fractional forward process. Suppose $f \in C^{2}\left(\mathbb{R}^{2}\right)$ and put $Y(t)=f(t, X(t))$. Then if $\frac{1}{2}<H<1$, we have

$$
d^{-} Y(t)=\frac{\partial f}{\partial t}(t, X(t)) d t+\frac{\partial f}{\partial x}(t, X(t)) d^{-} X(t)
$$


Proof. Let $0=t_{0}<t_{1}<\cdots<t_{N}=t$ be a partition of $[0, t]$. By using Taylor expansion, we get by equation (3.17)

$$
\begin{aligned}
Y(t)-Y(0) & =\sum_{j} Y\left(t_{j+1}\right)-Y\left(t_{j}\right) \\
& =\sum_{j} f\left(t_{j+1}, X\left(t_{j+1}\right)\right)-f\left(t_{j}, X\left(t_{j}\right)\right) \\
& =\sum_{j} \frac{\partial f}{\partial t}\left(t_{j}, X\left(t_{j}\right)\right) \Delta t_{j}+\sum_{j} \frac{\partial f}{\partial x}\left(t_{j}, X\left(t_{j}\right)\right) \Delta X\left(t_{j}\right) \\
& +\frac{1}{2} \sum_{j} \frac{\partial^{2} f}{\partial x^{2}}\left(t_{j}, X\left(t_{j}\right)\right)\left(\Delta X\left(t_{j}\right)\right)^{2}+\sum_{j} o\left(\left(\Delta t_{j}\right)^{2}\right)+o\left(\left(\Delta X\left(t_{j}\right)\right)^{2}\right) \\
& =\sum_{j} \frac{\partial f}{\partial t}\left(t_{j}, X\left(t_{j}\right)\right) \Delta t_{j}+\sum_{j} \int_{t_{j}}^{t_{j+1}} \frac{\partial f}{\partial x}\left(t_{j}, X\left(t_{j}\right)\right) d^{-} X_{t} \\
& +\frac{1}{2} \sum_{j} \frac{\partial^{2} f}{\partial x^{2}}\left(t_{j}, X\left(t_{j}\right)\right)\left(\Delta X\left(t_{j}\right)\right)^{2}+\sum_{j} o\left(\left(\Delta t_{j}\right)^{2}\right)+o\left(\left(\Delta X\left(t_{j}\right)\right)^{2}\right)
\end{aligned}
$$

where $\Delta X\left(t_{j}\right)=X\left(t_{j+1}\right)-X\left(t_{j}\right)$. Since $\frac{1}{2}<H<1$, the quadratic variation of the fractional Brownian motion is zero and we are left with the first terms of the sum above, which converges to $\int_{0}^{t} \frac{\partial f}{\partial s}(s, X(s)) d s+\int_{0}^{t} \frac{\partial f}{\partial x}(s, X(s)) d^{-} X(s)$.

Using the results of Theorem 3.7 and 3.10, we obtain a general Itô formula for functionals of Wick-Itô-Skorohod integrals with respect to the fractional Brownian motion when $\frac{1}{2}<H<1$. An Itô formula for $\frac{1}{2}<H<1$ has been already proved in [9] and in [4], but under more restrictive hypotheses. Here we provide a different proof under weaker assumptions. If $\frac{1}{2}<H<1$ this theorem extends Theorem 3.8 in [7]. A related result, obtained independently and by a different method, can be found in [11]. Moreover our results hold in a different setting.

Theorem 3.11 (Itô formula for the WIS-integral) Suppose $\frac{1}{2}<H<$ 1. Let $\gamma(s)$ be a measurable process such that $\int_{0}^{t}|\gamma(s)| d s<\infty$ a.s. for all $t \geq 0$, let $\psi(t)=\sum_{\alpha \in \mathcal{J}} c_{\alpha}(t) \mathcal{H}_{\alpha}(\omega)$ be càglàd, WIS-integrable and such that

$$
\sum_{\alpha \in \mathcal{J}} \sum_{i=1}^{\infty} \sum_{k=1}^{\infty}\left\|c_{\alpha}\right\|_{L^{2}([0, T])} \alpha_{i}\left(\alpha_{k}+1\right) \alpha !<\infty .
$$


Suppose that $M_{t} D_{t} \psi(s)$ is also WIS-integrable for almost all $t \in[0, T]$. Consider

$$
X(t)=x+\int_{0}^{t} \gamma(s) d s+\int_{0}^{t} \psi(s) d B^{(H)}(s), \quad t \in[0, T],
$$

or, in short-hand notation,

$$
d X(t)=\gamma(t) d t+\psi(t) d B^{(H)}(t), \quad X(0)=x .
$$

Suppose $X_{t}$ has a càdlàg version (Remark 3.12). Let $f \in C^{2}\left(\mathbb{R}^{2}\right)$ and put $Y(t)=f(t, X(t))$. Then on $[0, T]$

$d Y(t)=\frac{\partial f}{\partial t}(t, X(t)) d t+\frac{\partial f}{\partial x}(t, X(t)) d X(t)+\frac{\partial^{2} f}{\partial x^{2}}(t, X(t)) \psi(t) M_{t+} D_{t+} X(t) d t$,

and equivalently

$$
\begin{aligned}
d Y(t)= & \frac{\partial f}{\partial t}(t, X(t)) d t+\frac{\partial f}{\partial x}(t, X(t)) d X(t)+\frac{\partial^{2} f}{\partial x^{2}}(t, X(t)) \psi(t) M^{2}\left(\psi \chi_{[0, t]}\right)_{t} d t \\
& +\left[\frac{\partial^{2} f}{\partial x^{2}}(t, X(t)) \psi(t) \int_{0}^{t} M_{t}^{2} D_{t}^{(H)} \psi(u) d B^{(H)}(u)\right] d t
\end{aligned}
$$

where $M^{2}\left(\psi \chi_{[0, t]}\right)_{t}=M^{2}\left(\psi \chi_{[0, t]}\right)(t)$.

Proof. For simplicity we put $\alpha=0$. By Theorem 3.7 we have

$$
X(t)=\int_{0}^{t} \psi(s) d^{-} B^{(H)}(s)-\int_{0}^{t} M_{s+}^{2} D_{s+}^{(H)} \psi(s) d s
$$

We note that

$$
\begin{aligned}
\frac{1}{\epsilon} \int_{t}^{t+\epsilon} M_{s}^{2} D_{s}^{(H)}\left(f^{\prime}(X(t)) \psi(t)\right) d s=f^{\prime}(X(t)) \frac{1}{\epsilon} \int_{t}^{t+\epsilon} M_{s}^{2} D_{s}^{(H)} \psi(t) d s \\
+\psi(t) f^{\prime \prime}(X(t)) \frac{1}{\epsilon} \int_{t}^{t+\epsilon} M_{s}^{2} D_{s}^{(H)} X(t) d s
\end{aligned}
$$

Since $\psi \in \mathbb{L}_{1,2}^{(H)}$, the first term converges to $f^{\prime}(X(t)) M_{t+}^{2} D_{t+}^{(H)} \psi(t)$ as $\epsilon \rightarrow 0$. For the second term we restrict our attention to

$$
\begin{aligned}
\frac{1}{\epsilon} \int_{t}^{t+\epsilon} M_{s}^{2} D_{s}^{(H)} X(t) d s= & \underbrace{\frac{1}{\epsilon} \int_{t}^{t+\epsilon} \int_{0}^{t} M_{s}^{2} D_{s}^{(H)} \psi(u) d B^{(H)}(u) d s}_{a)} \\
& +\underbrace{\frac{1}{\epsilon} \int_{t}^{t+\epsilon} M_{s}^{2}\left(\psi \chi_{[0, t]}\right) d s}_{b)} .
\end{aligned}
$$


a) To study the convergence of the term a), we proceed as in Lemma 3.5. By using the chaos expansion we obtain

$$
\begin{aligned}
& \frac{1}{\epsilon} \int_{t}^{t+\epsilon} \int_{0}^{t} M_{s}^{2} D_{s}^{(H)} \psi(u) d B^{(H)}(u) d s= \\
& \sum_{\alpha \in \mathcal{J}} \sum_{i=1}^{\infty} \sum_{k=1}^{\infty}\left(c_{\alpha}, \xi_{k}\right)_{t} \frac{1}{\epsilon} \int_{t}^{t+\epsilon} \eta_{i}(s) d s \alpha_{i} \mathcal{H}_{\alpha-\epsilon^{(i)}+\epsilon^{(k)}}
\end{aligned}
$$

Put $\psi_{i, k, \alpha, \epsilon}:=\left(c_{\alpha}, \xi_{k}\right)_{t} \frac{1}{\epsilon} \int_{t}^{t+\epsilon} \eta_{i}(s) d s \alpha_{i} \mathcal{H}_{\alpha-\epsilon^{(i)}+\epsilon^{(k)}}$. Then

$$
\begin{array}{r}
\sum_{\alpha \in \mathcal{J}} \sum_{i=1}^{\infty} \sum_{k=1}^{\infty}\left\|\psi_{i, k, \alpha, \epsilon}\right\|_{L^{2}(\mathbb{P})}^{2}= \\
\sum_{\alpha \in \mathcal{J}} \sum_{i=1}^{\infty} \sum_{k=1}^{\infty}\left(c_{\alpha}, \xi_{k}\right)_{t}^{2}\left(\frac{1}{\epsilon} \int_{t}^{t+\epsilon} \eta_{i}(s) d s\right)^{2} \alpha_{i}\left(\alpha_{k}+1\right) \alpha ! \leq \\
{\left[\frac{(t+\epsilon)^{2 H}-t^{2 H}}{\epsilon}\right]^{2} \sum_{\alpha \in \mathcal{J}} \sum_{i=1}^{\infty} \sum_{k=1}^{\infty}\left\|c_{\alpha}\right\|_{L^{2}(0, T)}^{2}\left\|\xi_{k}\right\|_{L^{2}(0, T)}^{2} \alpha_{i}\left(\alpha_{k}+1\right) \alpha ! \leq} \\
{\left[\frac{(t+\epsilon)^{2 H}-t^{2 H}}{\epsilon}\right]^{2} \sum_{\alpha \in \mathcal{J}} \sum_{i=1}^{\infty} \sum_{k=1}^{\infty}\left\|c_{\alpha}\right\|_{L^{2}(0, T)}^{2} \alpha_{i}\left(\alpha_{k}+1\right) \alpha !,}
\end{array}
$$

where we have used that $\left\|\xi_{k}\right\|_{L^{2}(0, T)}^{2} \leq\left\|\xi_{k}\right\|_{L^{2}(0, T)}^{2}=1, \forall k=1,2, \cdots$. Since

$$
\frac{1}{\epsilon} \int_{t}^{t+\epsilon} \eta_{i}(s) d s \rightarrow \eta_{i}(t)
$$

and (3.23) holds, by Lemma 3.4 we conclude that

$$
\lim _{\epsilon \longrightarrow 0} \frac{1}{\epsilon} \int_{t}^{t+\epsilon} \int_{0}^{t} M_{s}^{2} D_{s}^{(H)} \psi(u) d B^{(H)}(u) d s=\int_{0}^{t} M_{t}^{2} D_{t}^{(H)} \psi(u) d B^{(H)}(u)
$$

in $L^{2}(\mathbb{P})$.

b) Since $\psi \in \mathbb{L}_{1,2}^{(H)}$, we have

$$
\frac{1}{\epsilon} \int_{t}^{t+\epsilon} M_{s}^{2}\left(\psi \chi_{[0, t]}\right) d s \longrightarrow M^{2}\left(\psi \chi_{[0, t]}\right)_{t}, \quad \text { a.e. and in } L^{2}(\mathbb{P}),
$$

where for the sake of simplicity we have put $M^{2}\left(\psi \chi_{[0, t]}\right)_{t}=M^{2}\left(\psi \chi_{[0, t]}\right)(t)$. Let $A_{t}=-\int_{0}^{t} M_{s+}^{2} D_{s+}^{(H)} \psi(s) d s$. Then by the Itô formula for forward integrals 
(Theorem 3.10) we obtain

$$
\begin{aligned}
d Y(t) & =f^{\prime}(X(t)) d^{-} X(t) \\
& =f^{\prime}(X(t)) d A_{t}+f^{\prime}(X(t)) d^{-} B^{(H)}(t) \\
& =-f^{\prime}(X(t)) M_{t+} D_{t+} \psi(t) d t+f^{\prime}(X(t)) \psi(t) d B^{(H)}(t) \\
& +\left[f^{\prime}(X(t)) M_{t+} D_{t+} \psi(t)+\psi(t) f^{\prime \prime}(X(t)) M_{t+} D_{t+} X(t)\right] d t \\
& =f^{\prime}(X(t)) d X(t)+f^{\prime \prime}(X(t)) \psi(t) M_{t+} D_{t+} X(t) d t
\end{aligned}
$$

and by (3.25) and (3.26) we can conclude that

$$
\begin{aligned}
d Y(t)= & f^{\prime}(X(t)) d X(t)+f^{\prime \prime}(X(t)) \psi(t) \int_{0}^{t} M_{t}^{2} D_{t}^{(H)} \psi(u) d B^{(H)}(u) d t \\
& +f^{\prime \prime}(X(t)) \psi(t) M^{2}\left(\psi \chi_{[0, t]}\right)_{t} d t .
\end{aligned}
$$

Note that all the integrands appearing in (3.27) are well-defined because $X_{t}$ is càdlàg.

Remark 3.12 Conditions under which the integral process admits a continuous modification are proved in [3] and [4].

Corollary 3.13 Assume that $\psi \in L_{H}^{2}(\mathbb{R}), \alpha=0$ and otherwise let $H, X, f, Y$ be as in Theorem 3.11. Then

$d Y(t)=\frac{\partial f}{\partial t}(t, X(t)) d t+\frac{\partial f}{\partial x}(t, X(t)) d X(t)+\frac{\partial^{2} f}{\partial x^{2}}(t, X(t)) \psi(t) M^{2}\left(\chi_{[0, t]} \psi\right)_{t} d t$

Remark 3.14 In the case when $\psi(s)$ is deterministic, a (different) Itô formula, valid for all $H \in(0,1)$ and for all $x$-entire functions $f(t, x)$ of order 2, has been obtained in Theorem 11.1 of [15].

\section{Examples}

\subsection{A special case}

In [5] and [7] an Itô formula for the case when $Y(t)=f\left(B^{(H)}(t)\right)$ is provided, valid for all $H \in(0,1)$. We recall here that formula

$$
d Y(t)=f^{\prime}(X(t)) d X(t)+H t^{2 H-1} f^{\prime \prime}(X(t)) \psi(t) d t
$$


We now show that if $H>\frac{1}{2}$ then (3.20) and (4.1) coincide in this case.

Proposition 4.1 For every $H \in(0,1)$ we have

$$
M_{t+} D_{t+} B^{(H)}(t)=H t^{2 H-1}, \quad t \geq 0 .
$$

Proof. Let $t \geq 0$. We recall that $D_{t}^{(H)} B^{(H)}(u)=\chi_{[0, u)}(t)$. Hence we need to prove that

$$
\begin{aligned}
M_{t+} D_{t+} B^{(H)}(t) & =\lim _{s \longrightarrow t^{+}} \frac{1}{\epsilon} \int_{t}^{t+\epsilon} M_{s}^{2} D_{s}^{(H)} B^{(H)}(t) d s \\
& =\left[M_{t}^{2} \chi_{[0, u)}(t)\right]_{u=t}=H t^{2 H-1}
\end{aligned}
$$

We consider $\psi(u)=\int_{\mathbb{R}}\left(M_{t} \chi_{[0, u)}(t)\right)^{2} d t$. Since, by [10], we have that $\psi(u)=$ $u^{2 H}$, we only need to show that $\psi^{\prime}(u)=2\left[M_{t}^{2} \chi_{[0, u)}(t)\right]_{t=u}$. We rewrite $\psi(u)$ as follows

$$
\begin{aligned}
\psi(u) & =\int_{\mathbb{R}}\left(M_{t} \chi_{[0, u)}(t)\right)^{2} d t \\
& =\int_{\mathbb{R}} \chi_{[0, u)}(t) M_{t}^{2} \chi_{[0, u)}(t) d t \\
& =\int_{0}^{u} M_{t}^{2} \chi_{[0, u)}(t) d t
\end{aligned}
$$

by using the properties of the operator $M$. We compute

$$
\begin{aligned}
& \frac{\psi(u+\epsilon)-\psi(u)}{\epsilon} \\
& =\frac{1}{\epsilon}\left(\int_{0}^{u+\epsilon} M_{t}^{2} \chi_{[0, u+\epsilon]}(t) d t-\int_{0}^{u} M_{t}^{2} \chi_{[0, u)}(t) d t\right) \\
& =\frac{1}{\epsilon}\left(\int_{u}^{u+\epsilon} M_{t}^{2} \chi_{[0, u+\epsilon]}(t) d t+\int_{0}^{u}\left[M_{t}^{2} \chi_{[0, u+\epsilon]}(t)-M_{t}^{2} \chi_{[0, u)}(t)\right] d t\right)
\end{aligned}
$$

by adding and subtracting $\int_{0}^{u} M_{t}^{2} \chi_{[0, u+\epsilon]}(t) d t$. Since the operator $M$ transforms $\chi_{[0, u)}(t)$ into a continuous function, we obtain

1. $\int_{u}^{u+\epsilon} M_{t}^{2} \chi_{[0, u+\epsilon]}(t) d t=\left[M_{t}^{2} \chi_{[0, u+\epsilon]}(t)\right]_{t=\xi_{\epsilon}} \epsilon$, where $u<\xi_{\epsilon}<u+\epsilon$. By writing

$$
\left[M_{t}^{2} \chi_{[0, u+\epsilon]}(t)\right]_{t=\xi_{\epsilon}}=\left[M_{t}^{2}\left(\chi_{[0, u+\epsilon]}-\chi_{[0, u)}\right)(t)\right]_{t=\xi_{\epsilon}}+\left[M_{t}^{2} \chi_{[0, u)}(t)\right]_{t=\xi_{\epsilon}}
$$

we obtain that, when taking the limit as $\epsilon \longrightarrow 0$, the first term goes to zero, while the second term converges to $\left[M_{t}^{2} \chi_{[0, u)}(t)\right]_{t=u}$ since $\xi_{\epsilon} \longrightarrow u$ when $\epsilon \longrightarrow 0$. 
2. We have that

$$
\begin{array}{r}
\frac{1}{\epsilon} \int_{0}^{u}\left[M_{t}^{2} \chi_{[0, u+\epsilon]}(t) d t-M_{t}^{2} \chi_{[0, u)}(t)\right] d t= \\
\frac{1}{\epsilon} \int_{0}^{u} M_{t}^{2}\left[\chi_{(u, u+\epsilon]}(t)\right] d t= \\
\frac{1}{\epsilon} \int_{0}^{T} \chi_{[0, u)}(t)\left(M_{t}^{2}\left[\chi_{(u, u+\epsilon]}(t)\right] d t=\right. \\
\frac{1}{\epsilon} \int_{u}^{u+\epsilon} M_{t}^{2}\left[\chi_{[0, u)}(t)\right] d t
\end{array}
$$

converges to $\left[M_{t}^{2} \chi_{[0, u)}(t)\right]_{t=u}$ as $\epsilon \longrightarrow 0$.

Hence

$$
\psi^{\prime}(u)=\lim _{\epsilon \longrightarrow 0} \frac{\psi(u+\epsilon)-\psi(u)}{\epsilon}=2\left[M_{t}^{2} \chi_{[0, u)}(t)\right]_{t=u}
$$

i.e. the equality $\left[M_{t}^{2} \chi_{[0, u)}(t)\right]_{t=u}=H u^{2 H-1}$ holds for every $H \in(0,1)$.

\subsection{An integration by parts formula}

Let $\psi(s)=\psi(s, \omega) \in \mathbb{L}_{1,2}^{(H)}$ be $d B^{(H)}$-integrable and define

$$
X(t)=\int_{0}^{t} \psi(s) d B^{(H)}(s)
$$

and

$$
Y(t)=X^{2}(t)
$$

By (3.25) and (3.26) we have

$$
M_{t+} D_{t+} X(t)=\int_{0}^{t} M_{t} D_{t} \psi(s) d B^{(H)}(s)+M^{2}\left(\psi \chi_{[0, t]}\right)_{t},
$$

where $M^{2}\left(\psi \chi_{[0, t]}\right)_{t}=M^{2}\left(\psi \chi_{[0, t]}\right)(t)$. Then by Theorem 3.11 and by Proposition 2.12 we have

$$
d Y(t)=2 X(t) d X(t)+2 \psi(t)\left(\int_{0}^{t} M_{t} D_{t} \psi(s) d B^{(H)}(s)+M^{2}\left(\psi \chi_{[0, t]}\right)_{t}\right) d t
$$

In particular, if $\psi \in L_{H}^{2}(\mathbb{R})$, we get

$$
d Y(t)=2 X(t) d X(t)+2 \psi(t) M^{2}\left(\psi \chi_{[0, t]}\right)_{t} d t
$$

By using that $X_{1} X_{2}=\frac{1}{2}\left[\left(X_{1}+X_{2}\right)^{2}-X_{1}^{2}-X_{2}^{2}\right]$ this gives the following product rule: 
Proposition 4.2 (Product rule) Suppose $\psi_{1}, \psi_{2} \in L_{H}^{2}(\mathbb{R})$ and define

$$
X_{i}(t)=\int_{0}^{t} \psi_{i}(s) d B^{(H)}(s) ; \quad i=1,2
$$

and

$$
Y(t)=X_{1}(t) X_{2}(t)
$$

Then

$$
\begin{aligned}
d Y(t) & =X_{1}(t) d X_{2}(t)+X_{2}(t) d X_{1}(t) \\
& +\left\{\psi_{1}(t) M^{2}\left(\psi_{2} \chi_{[0, t]}\right)_{t}+\psi_{2}(t) M^{2}\left(\psi_{1} \chi_{[0, t]}\right)_{t}\right\} d t
\end{aligned}
$$

Corollary 4.3 (Integration by parts) Let $X_{i}(t), i=1,2$, be as in Proposition 4.2. Then

$$
\begin{aligned}
\int_{0}^{t} X_{1}(s) d X_{2}(s) & =X_{1}(t) X_{2}(t)-\int_{0}^{t} X_{2}(s) d X_{1}(s) \\
& -\int_{0}^{t}\left\{\psi_{1}(s) M^{2}\left(\psi_{2} \chi_{[0, s]}\right)_{s}+\psi_{2}(s) M^{2}\left(\psi_{1} \chi_{[0, s]}\right)_{s}\right\} d s .
\end{aligned}
$$

\section{References}

[1] K. Aase, B. Øksendal, N.Privault, and J. Ubøe: White noise generalizations of the Clark-Haussmann-Ocone theorem with application to mathematical finance. Finance and Stochastics 4 (2000), 465-496.

[2] E. Alòs, A. León, D. Nualart: Stratonovich stochastic calculus with respect to to fractional Brownian motion with Hurst parameter less than 1/2. Taiwanesse Journal of Mathematics 5, 609-632, 2001.

[3] E. Alòs, O. Mazet, D. Nualart: Stochastic calculus with respect to Gaussian processes. Ann. Probab. 29, 766-801, 2000.

[4] E.Alòs, D.Nualart: Stochastic integration with respect to the fractional Brownian motion. Stochastics and Stochastics Reports (2004), to appear.

[5] C.Bender: An Itô formula for generalized functionals of a fractional Brownian motion with arbitrary Hurst parameter. Stoch.Proc. Appl. 104 (1), 81-106, 2003.

[6] F.Biagini, Y.Hu, B.Øksendal,T.Zhang: Fractional Brownian Motion and Applications. (Forthcoming book, Springer) 
[7] F.Biagini, B.Øksendal, A.Sulem, N.Wallner: An introduction to white noise theory and Malliavin calculus for fractional Brownian motion, The Proceedings of the Royal Society, 460, (2004), 347-372. .

[8] Cheridito P., Nualart, D.: Stochastic integral of divergence type with respect to the fractional Brownian motion with Hurst parameter $H<\frac{1}{2}$. Ann. Inst. H. Poincaré Probab. Statist. 41, 1049-1081, 2005.

[9] T.E. Duncan, Y. Hu and B. Pasik-Duncan: Stochastic calculus for fractional Brownian motion. I. Theory. SIAM J. Control Optim. 38 (2000), $582-612$.

[10] R. J. Elliott and J. Van der Hoek: A general fractional white noise theory and applications to finance.Mathematical Finance, 13 (2003), 301-330.

[11] R. J. Elliott and J. Van der Hoek: A basic lemma for stochastic integrals and Itô formulas for processes driven by fractional Brownian motion. Working paper 2004.

[12] M.Gradinaru, I.Nourdin, F.Russo, P.Vallois: $m$-order integrals and generalized Itô's formula; the case of a fractional Brownian motion with any index. Ann. Inst. H. Poincaré Probab. Statist. 41, no. 4, 781-806, 2005.

[13] T. Hida, H. -H Kuo, J. Potthoff and L. Streit: White Noise Analysis. Kluwer, 1993.

[14] H. Holden, B. Øksendal, J. Ubøe and T. Zhang: Stochastic Partial Differential Equations. Birkhäuser 1996.

[15] Y. Hu: Integral transformations and anticipative calculus for fractional Brownian motions. Memoirs of the American Mathematical Society 825, 2005.

[16] Y. Hu and B. Øksendal: Fractional white noise calculus and applications to finance. Inf.Dim.Anal.Quant.Probab. 6 (2003), 1-32.

[17] H. -H.Kuo: White Noise Distribution Theory. CRC Press 1996.

[18] Y.Mishura: Fractional stochastic integration and Black-Scholes equation for fractional Brownian model with stochastic volatility. Stoch. Stoch. Reports, 76, 4, 363-381, 2004.

[19] D.Nualart: Stochastic integration with respect to fractional Brownian motion 
and applications. Stochastic Models (Mexico City,2002), Contemp.Math. 336 Americn.Math.Soc., Providence, RI, 3-39, 2003.

[20] D. Nualart, E. Pardoux: Stochastic calculus with anticipative integrands. Prob. Th. Rel. Fields 78, 535-581, 1988.

[21] B. Øksendal: Fractional Brownian motion in finance. In B. S. Jensen and T. Palokangas (editors): Stochastic Economic Dynamics, Cambridge Univ. Press (to appear).

[22] L.C.G. Rogers and D. Williams: Diffusions, Markov Processes, and Martingales, Volume 1: Foundations, Cambridge University Press, 2000.

[23] F.Russo and P.Vallois: Stochastic calculus with respect to continuous finite quadratic variation processes, Stochastics and Stochastics Reports 70, (2000), 1-40.

[24] A.N.Shiryaev: Essentials of Stochastic Finance: Facts, Models and Theory. World Scientific 1999.

[25] S. Thangavelu: Lectures of Hermite and Laguerre Expansions. Princeton University Press, 1993.

[26] M.Zähle: Integration with respect to fractal functions and stochastic calculus II. Math.Nachr., 225 (2001), 145-183.

[27] M.Zähle: Forward integrals and stochastic differential equations. Progress in Probability, Vol.52, (2002), 293-302. 